H. Imai

Nagoya Math. J.

Vol. 59 (1975), $45-58$

\title{
THE VALUE DISTRIBUTION OF HARMONIC MAPPINGS BETWEEN RIEMANNIAN $N$-SPACES
}

\author{
HIDEO IMAI
}

We are concerned with the value distribution of a mapping of an open Riemannian $n$-space ( $n \geqq 3$ ) into a Riemannian $n$-space. The value distribution theory of an analytic mapping of Riemann surfaces was initiated by S. S. Chern [1] and developed mainly by L. Sario [8], [9], [10], [11], and then by $\mathrm{H}$. Wu [14], [15]. The most crucial part in Sario's theory is the introduction of a kernel function on an arbitrary Riemann surface to describe appropriately the proximity of two points. His method indicates that the potential theoretic method is one of the powerful methods in the value distribution theory.

Our main object is to generalize the first main theorem to the higher dimensional Riemannian spaces according to Sario's method. But in view of the intrinsic restriction, we need to confine ourselves to a mapping which does not destroy the harmonic structure. For this purpose we introduce the notion of a harmonic mapping which was first considered by C. Constantinescu-A. Cornea in the theory of harmonic spaces ([2]). Roughly speaking, it is a mapping preserving harmonic functions. We will see that the first main theorem is valid for an arbitrary harmonic mapping.

In our present paper we will first introduce the harmonic mapping between Riemannian spaces. We will show that it is a $C^{2}$-mapping and a local isometry except for the set of singular points of the mapping (Lemmas 1 and 4). To construct the characteristic function, the function obtained from Sario's kernel by applying Laplacian plays the role of the volume element on Riemannian spaces. In section 4 we will see that the total volume of any Riemannian spaces (which is either open

Received March 5, 1973.

Revised October 17, 1974.

The author wishes to express his indebtness to Professor Mitsuru Nakai for his valuable advice. 
or closed) is equal to 2 with respect to this volume element. Finally we will obtain the first main theorem for harmonic mappings which states that the counting number of the pre-image of a non-singular point $a$ and the mean proximity of the image of the relative boundary to a point $a$ is determined only by the given region of the domain space and the given harmonic mappings.

1. Let $R$ be a Riemannian $n$-space ( $n \geqq 3$ ), i.e., a connected, separable and orientable $n$-dimensional $(n \geqq 3) C^{\infty}$-manifold with a $C^{\infty}$-metric tensor $g_{i j}$. A relatively compact subregion $\Omega$ of $R$, the boundary of which are piecewise $C^{\infty}$, is called regular. A sequence $\left\{\Omega_{n}\right\}_{n=1}^{\infty}$ of regular regions with $\bar{\Omega}_{n} \subset \Omega_{n+1}$ and $R=\cup \Omega_{n}$ is called a regular exhaustion of $R$. For an open set $\omega$ of $R, H(\omega)$ stands for the class of harmonic functions on $\omega$ and $H^{c}(\omega)$ for the subclass of $H(\omega)$, consisting of functions which can be continuously extendable to the closure $\bar{\omega}$ of $\omega$. Denote by $g_{V}(x, \zeta)$ Green's function of $V$ with its pole at $\zeta$ and with the normalization $-\int_{\partial V} * d g(x, \zeta)=1$ for any parametric ball $V$ at $\zeta$.

Let $S$ be another Riemannian $n$-space with a $C^{\infty}$-metric tensor $\bar{g}_{k \ell}$. The mapping $f: R \rightarrow S$ is called an isometry if $f$ is a diffeomorphism of $R$ onto $f(R)$ and $g^{i j}(x)=\bar{g}^{k \ell}(f(x))$ for each $x$ in $R$. For each point $x$ in $R$, if there exist open neighbourhoods $U$ of $x$ and $V$ of $y=f(x)$ such that $f$ is an isometry of $U$ onto $V, f$ is called a local isometry. As usual, a point $x_{0} \in R$ is a singular point of $f$ if $\operatorname{det}\left(\frac{\partial y^{j}}{\partial x^{i}}\right)=0$ at $x=x_{0}$, where $x=\left(x^{1}, \cdots, x^{n}\right)$ is a local parameter about $x$ and $y^{j}=f^{j}(x)$ with $f(x)=\left(f^{1}(x), \cdots, f^{n}(x)\right)$. For the singular point $x_{0}, y_{0}=f\left(x_{0}\right)$ is called a singular value of $f$. Similarly, for any $C^{1}$-function $\varphi$ in $R$, a point $x_{0}$ in $R$ is a critical point of $\varphi$ if $\left(\frac{\partial \varphi}{\partial x^{1}}, \cdots, \frac{\partial \varphi}{\partial x^{n}}\right)=0$ at $x=x_{0}$, and for the critical point $x_{0}$ of $\varphi, \varphi\left(x_{0}\right)$ is called a critical value of $\varphi$.

2. Let $f$ be a continuous mapping of $R$ to $S$. A mapping $f$ is called harmonic if for any point $x$ in $R$, any neighbourhood $V$ of $y=$ $f(x)$ in $f(R)$, and any harmonic function $u_{x}$ in $V, u_{x} \circ f$ is harmonic on the pre-image $f^{-1}(V)$ of $V$ under $f$. This definition is a version of the harmonic mapping in the theory of harmonic spaces which was introduced by C. Constantinescu-A. Cornea [2]. We will consider the properties of harmonic mappings according to those in the theory of harmonic spaces. First we will show that $f$ becomes automorphically a $C^{2}$-mapping. 
LEMma 1. Let $f$ be a harmonic mapping of $R$ to $S$. Then $f$ is a $C^{2}$-mapping.

Proof. It suffices to show that $f$ is locally $C^{2}$-mapping. For each point $x_{0} \in R$, let $y_{0}=f\left(x_{0}\right)$. Denote by $V_{0}$ and $\Omega$ the concentric parametric balls about $y_{0}$ in $S$ such that $V_{0} \not \subset \Omega$ and by $g_{\Omega}\left(y, y_{0}\right)$ the Green's function of $\Omega$ with a pole at $y_{0}$. Without loss of generality, we may assume that there are no critical points of $g_{\Omega}\left(y, y_{0}\right)$ in $\Omega-\left\{y_{0}\right\}$, because of the compactness of $\bar{\Omega}$ and of Sard's theorem. Let $\alpha_{1}, \alpha_{2}, \cdots, \alpha_{n}$ be real numbers such that $0<\alpha_{1}<\cdots<\alpha_{n}<\min _{\partial V_{0}} g_{\Omega}\left(y, y_{0}\right)$ and $\eta_{k}=\left(\eta_{1}^{k}, \cdots, \eta_{n}^{k}\right)$ be a point in the hypersurface $\left\{g_{\Omega}\left(y, y_{0}\right)=\alpha_{k}\right\}$ with $k=1,2, \cdots, n$. Since $g_{s}\left(\eta_{k}, y\right)=O\left(\rho\left(\eta_{k}, y\right)^{2-n}\right)$ with $\rho\left(\eta_{k}, y\right)^{2}=g_{i j}\left(\eta_{i}^{k}-y_{i}^{0}\right)\left(\eta_{j}^{k}-y_{j}^{0}\right)$ and $y^{0}=\left(y_{1}^{0}\right.$, $\left.\cdots, y_{n}^{0}\right)$, we may choose $\eta_{k}$ such that the $k$-th coordinate of $\eta_{k}$ is the only non-zero coordinate. Then, since $\operatorname{grad} g_{\Omega}\left(y, y_{0}\right) \neq 0$ at $y=\eta_{k}$, the $k$-th component of $\left(\frac{\partial}{\partial y_{1}} g_{\Omega}\left(y, y_{0}\right), \cdots, \frac{\partial}{\partial y_{n}} g_{\Omega}\left(y, y_{0}\right)\right)$ at $y=\eta_{k}$ is the only non-zero component, where $\left(y_{1}, \cdots, y_{n}\right)$ is a local parameter of the ball $\Omega$. By the symmetry of Green's function, $g_{\Omega}\left(\eta_{k}, y_{0}\right)=g_{\Omega}\left(y_{0}, \eta_{k}\right)$ and then the components of $\left(\frac{\partial}{\partial y_{1}} g_{\Omega}\left(y_{0}, \eta_{k}\right), \cdots, \frac{\partial}{\partial y_{n}} g_{\Omega}\left(y_{0}, \eta_{k}\right)\right)$ at $y=\eta_{k}(k=1,2$, $\cdots, n)$ are not zero only at the $k$-th component. We set $u_{k}(y)=g_{\Omega}\left(y, \eta_{k}\right)$ for $k=1,2, \cdots, n$. Clearly $H\left(V_{0}\right) \supset\left\{u_{k}\right\}_{k=1}^{n}$ and $\operatorname{det}\left(\frac{\partial}{\partial y_{i}} u_{j}(y)\right)_{\substack{1 \leqq i \leqq n \\ 1 \leqq j \leqq n}} \neq 0$ at $y=y_{0}$. Let $V$ be a connected neighbourhood of $y_{0}$ such that $\operatorname{det}\left(\frac{\partial}{\partial y_{i}} u_{j}(y)\right) \neq 0$ in $f(R)$ and $U$ be a connected component of $f^{-1}(V)$. Then, by the hypothesis, $u_{k} \circ f \in H(U)$ and hence $u_{k} \circ f(k=1,2, \cdots, n)$ is a $C^{2}$-function in $U$. Since, by the construction of $\left\{u_{k}\right\}_{k=1}^{n},\left(u_{1}, \cdots, u_{n}\right)$ serves as a local parameter in $V, f$ is a $C^{2}$-mapping in $U$.

In order to show the global property of a harmonic mapping, we need the following approximation Lemma, the original form of which can be found in [6].

LEMMA 2 ([6]). Let $G$ be a Riemannian space and $\omega$ be an open subset of $G$. For any $u \in H(\omega)$ and any compact subset $K$ of $\omega, u$ can be uniformly approximated by functions in $H(G)$ on $K$ if and only if the complement $\complement \omega$ of $\omega$ does not contain connected components.

Lemma 3. Let $f$ be a $C^{2}$-mapping of $R$ to $S$. Then, $f$ is a 
harmonic mapping if and only if $u \cdot f$ if harmonic on $R$ for every $u \in$ $H(f(R))$.

Proof. Since the "if part" is trivial, we will show the "only if part". Let $x \in R$ be any point and $V$ be any neighbourhood of $y=f(x)$ in $f(R)$. Without loss of generality, we may assume that the complement $\lceil V$ of $V$ has no connected compact components in $S$. Indeed otherwise, we can choose an open covering $\left\{V_{j}\right\}$ of $V$ in $f(R)$ such that each $V_{j}$ has no connected compact components in $S$ and the point $y=f(x)$ is contained in some $V_{j}$. Let $u_{x}$ be any function in $H(V)$ and $\left\{V_{n}\right\}_{n=1}^{\infty}$ be a regular exhaustion of $V$ such that $V_{n} \subset \bar{V}_{n} \subset V_{n+1}$ for each $n$ and $V=\bigcup_{n=1}^{\infty} V_{n}$. For every $n \geqq 2$, we denote by $h_{n}$ a harmonic function in $S$ with the property that $\left|u_{x}-h_{n}\right|<1 / n$ on $\bar{V}_{n-1}$. This procedure is possible by Lemma 2. Then, by the hypothesis, $h_{n} \cdot f \in H(R)$ and $\left|u_{x} \circ f-h_{n} \circ f\right|<1 / n$ on $f^{-1}\left(\bar{V}_{n-1}\right)$ for each $n$. Hence taking the limit, $u_{x} \circ f$ coincides with some function $h \in H(R)$ on $f^{-1}(V)$. Therefore we have $u_{x} \circ f \in H\left(f^{-1}(V)\right)$.

LEMMa 4. For any harmonic mapping $f$ of $R$ to $S, f$ is a local isometry except for the set of singular points of $f$.

Proof. Let $x_{0}$ be any non-singular point of $f$. Then there exist open neighbourhoods $U$ of $x_{0}$ and $V$ of $y_{0}=f\left(x_{0}\right)$ such that $f$ is a bijective $C^{2}$-mapping of $U$ onto $V$. Since, by the hypothesis, $f$ preserves harmonic functions, it is an isometry of $U$ onto $V$ (c.f. [3, p 387]).

The following properties of harmonic mappings are known from the general theory of harmonic spaces.

LEMMA 5. Let $f$ be a harmonic mapping of $R$ to $S$. If a subset $A$ of $f(R)$ is polar, $f^{-1}(A)$ is also a polar set. If $s^{\prime}$ is a superharmonic function in an open subset $G$ of $f(R)$, then $s^{\prime} \cdot f$ is superharmonic in $f^{-1}(G)$.

COROLLARY 1. Under a harmonic mapping $f, f^{-1}\left(y_{0}\right)$ is the set of $n$-dimensional Newtonian capacity zero for each singular value $y_{0} \in f(R)$, and $f^{-1}(y)$ is at most countable point set for each non-singular value $y \in f(R)$.

Proof. Let $y_{0}$ be a singular value of $f$ and $V$ be a neighbourhood of $y_{0}$ in $f(R)$. Then there exists the Green's function $g_{V}\left(y, y_{0}\right)$ of $V$. 
By Lemma 5, $g_{V}\left(f(x), y_{0}\right)$ is superharmonic in $f^{-1}(V)$ and $g_{V}\left(f(x), y_{0}\right)=$ $\infty$ on $f^{-1}\left(y_{0}\right)$. Therefore the set $f^{-1}\left(y_{0}\right)$ is polar and it is a set of $n$ dimensional Newtonian capacity zero. The second part is clear by Lemma 4 and the $\sigma$-compactness of $R$.

A typical example of a harmonic mapping is the projection of the covering Riemannian space. In this case the harmonic mapping has no singular points.

3. We will recall the Sario kernel on $S$ and then consider the properties of the composed Sario's kernel under a harmonic mapping $f$. These are the basic tool in the value distribution theory by Sario's method.

In $S$ we take the fixed point $y_{j}(j=0,1)$ and then the disjoint parametric ball $V_{j}(j=0,1)$ with the center at $y_{j}$. Let $g_{V_{j}}\left(y, y_{j}\right)(j=0,1)$ be the corresponding Green's function. Usign Sario's principal operator $L_{1}$ (c.f. [7]), construct the harmonic function $t_{0}(y)=t_{0}\left(y, y_{0}, y_{1}\right)$ on $S-$ $\left\{y_{0}, y_{1}\right\}$ such that $t_{0}(y)-g_{V_{0}}\left(y, y_{0}\right)$ and $t_{0}(y)+g_{V_{1}}\left(y, y_{1}\right)$ are harmonic in $V_{0}$ and $V_{1}$ respectively, and that $t_{0}=L_{1} t_{0}$ in a neighbourhood of the ideal boundary. Normalize $t_{0}(y)$ in such a way that $t_{0}(y)-g_{V_{0}}\left(y, y_{0}\right) \rightarrow 0$ as $y \rightarrow y_{0}$ in $V_{0}$. Then the functions $s_{0}(y)=\log \left(1+e^{t_{0}(x)}\right)$ and $s_{0}(y)-g_{V_{0}}\left(y, y_{0}\right)$ are finitely continuous in $S-\left\{y_{0}\right\}$ and $V_{0}$ respectively. For an arbitrary point $a$ in $S-\left\{y_{0}\right\}$, we construct the function $t(y, a)=t\left(y, a, y_{0}\right)$ in a similar manner to $t_{0}\left(y, y_{0}, y_{1}\right)$ except for the normalization: $t(y, a)+g_{V_{0}}\left(y, y_{0}\right)$ $\rightarrow s_{0}(a)$ as $y \rightarrow y_{0}$ in $V_{0}$. For convenience, in these constructions we always take $y_{0}, y_{1}$ and $a$ from the set of non-singular values of $f$.

Let $s_{1}(y, a)=s_{0}(y)+t(y, a)$ and make $s_{1}\left(y, y_{0}\right)=s_{0}\left(y_{0}\right)$. Then $s_{1}(y, a)$ is bounded from below. We set $s(y, a)=s_{1}(y, a)+c$, where the constant $c$ is choosen so as to have $s(y, a)>0$ on $S \times S$. The function $s(y, a)$ is called Sario's kernel on $S$ which is a symmetric kernel. The details for Sario's kernel may be found in [4], [7] and [13]. Sario's kernel has the following properties;

LEMMA 6 ([4]). On $S-\left\{y_{0}, y_{1}\right\}$

$$
\Delta_{y} s_{0}(y)=e^{t_{0}(y)}\left(1+e^{t_{0}(y)}\right)^{-2}\left|\operatorname{grad} t_{0}(y)\right|^{2}
$$

holds, and hence $\Delta_{y} s_{0}(y)$ is non-negative there.

Lemma 7 ([4]). Sario's kernel $s(y, a)$ is jointly continuous on $S \times S$. 
For every regular region $\Omega^{\prime}$ of $S$ which contains the point $a$, the decomposition

$$
s(y, a)=g_{\Omega^{\prime}}(y, a)+v_{\Omega^{\prime}}(y, a)
$$

is given, where $g_{\Omega^{\prime}}$ is the Green's function on $\Omega^{\prime}$ and $v_{\Omega^{\prime}}$ is a finitely continuous function on $\Omega^{\prime} \times \Omega^{\prime}$.

Let $f: R \rightarrow S$ be a harmonic mapping and $\left\{a_{j}\right\}$ be the pre-image of $a$ under $f$. Since $a$ is a non-singular value of $f,\left\{a_{j}\right\}$ is a totally disconnected point set in $R$. Choose one $a_{j}$ in $\left\{a_{j}\right\}$ and denote by $U$ and $V$ the isometric neighbourhoods of $a_{j}$ and $a$ under $f$, respectively. $\alpha$ stands for the boundary of $U$.

LEMMA 8. For an arbitrary harmonic mapping $f$ and each $a_{j}$ in $\left\{a_{j}\right\}$

$$
\int_{\alpha} * d s\left(f(x), f\left(a_{j}\right)\right)=\int_{\alpha} * d g_{U}\left(x, a_{j}\right)=\int_{\alpha} * d g_{V}\left(f(x), f\left(a_{j}\right)\right)=-1
$$

is valid and hence, for any $\varphi \in H^{C}(\bar{U})$,

$$
\varphi\left(a_{j}\right)=\int_{\alpha} \varphi(x) * d g_{V}\left(f(x), f\left(a_{j}\right)\right) .
$$

Proof. Since the harmonic structure is invariant under isometries, the property of Green's function implies that $g_{U}\left(x, a_{j}\right)=g_{V}\left(f(x), f\left(a_{j}\right)\right)$ and $\int_{\alpha} * d g\left(x, a_{j}\right)=\int_{\alpha} * d g\left(f(x), f\left(a_{j}\right)\right)=-1$. Also, by Lemma 7 , $\int_{\alpha} * d s\left(f(x), f\left(a_{j}\right)\right)=\int_{\alpha} * d g_{V}\left(f(x), f\left(a_{j}\right)\right)$. Thus we obtain the first part. The second part follows immediately by Green's formula.

The composed function $s_{0}(f(x))$ has the following properties;

LEMMA 9. On $R-f^{-1}\left(y_{0}\right) \cup f^{-1}\left(y_{1}\right)$

$$
\Delta_{x} s_{0}(f(x))=e^{t_{0}(f(x))}\left(1+e^{t_{0}(f(x))}\right)^{-2}\left|\operatorname{grad}_{x} t_{0}(f(x))\right|^{2}
$$

holds, where $\left|\operatorname{grad}_{x} t_{0}(f(x))\right|^{2}=g^{i j}(x) \frac{\partial t_{0} f}{\partial x^{i}} \frac{\partial t_{0} f}{\partial x^{j}}(x)$. Consequently $\Delta_{x} s_{0}(f(x))$ is subharmonic there.

Proof. For any point $x=\left(x^{1}, x^{2}, \cdots, x^{n}\right)$ in $R-f^{-1}\left(y_{0}\right) \cup f^{-1}\left(y_{1}\right)$,

$$
\frac{\partial}{\partial x^{i}} s_{0}(f(x))=e^{t_{0}(f(x))}\left(1+e^{t_{0}(f(x))}\right)^{-1} \frac{\partial}{\partial x^{i}} t_{0}(f(x)) \text {, }
$$




$$
\begin{aligned}
& \left(\frac{\partial}{\partial x^{i}} g^{i j}+g^{i j} \frac{\partial}{\partial x^{i}} \log \sqrt{g}\right) \frac{\partial}{\partial x^{j}} s_{0}(f(x)) \\
& \quad=e^{t_{0}(f(x))}\left(1+e^{t_{0}(f(x))}\right)^{-1}\left(\frac{\partial}{\partial x^{i}} g^{i j}+g^{i j} \frac{\partial}{\partial x^{i}} \log \sqrt{g}\right) \frac{\partial}{\partial x^{j}} t_{0}(f(x)),
\end{aligned}
$$

and

$$
\begin{aligned}
g^{i j} \frac{\partial^{2}}{\partial x^{i} \partial x^{j}} s_{0}(f(x))= & e^{t_{0}(f(x))}\left(1+e^{t_{0}(f(x))}\right)^{-2}\left|\operatorname{grad}_{x} t_{0}(f(x))\right|^{2} \\
& +e^{t_{0}(f(x))}\left(1+e^{t_{0}(f(x))}\right)^{-1} g^{i j} \frac{\partial^{2}}{\partial x^{i} \partial x^{j}} t_{0}(f(x)) .
\end{aligned}
$$

Since $\Delta_{x} t_{0}(f(x))=0$ on $R-f^{-1}\left(y_{0}\right) \cup f^{-1}\left(y_{1}\right)$, we obtain

$$
\Delta_{x} s_{0}(f(x))=e^{t_{0}(f(x))}\left(1+e^{t_{0}(f(x))}\right)^{-2}\left|\operatorname{grad}_{x} t_{0}(f(x))\right|^{2} .
$$

4. We will construct the volume element of $S$ which is necessary in order to define the characteristic function. Let $\lambda^{2}(y) d V_{y}$ be the $n$ form on $S-\left\{y_{0}, y_{1}\right\}$ defined by

$$
\lambda^{2}(y)=e^{t_{0}(y)}\left(1+e^{t_{0}(y)}\right)^{-2}\left|\operatorname{grad}_{y} t_{0}(y)\right|^{2},
$$

with $\lambda(y) \geqq 0$ and $d V_{y}$ be the volume element on $S$, i.e., locally $d V_{y}=$ $n \omega_{n} r^{n} d r$ with $\omega_{n} r^{n}$ the volume of the ball of the radius $r$. Evidently $\lambda^{2}(y) d V_{y}$ is non-negative and finite on $S-\left\{y_{0}, y_{1}\right\}$.

LEMMA 10. As $r=\left|y-y_{j}\right| \rightarrow 0(j=0,1)$

$$
\lambda^{2}(y) \sim \exp \left(-r^{2-n}\right)
$$

holds. Hence $\lambda^{2}(y)$ is non-negative and finite on $S$ and the set of zero points of $\lambda^{2}(y)$ consists of $y_{0}, y_{1}$, and the critical points of $t_{0}(y)$.

Proof. The points that we need to be checked are $y_{0}$ and $y_{1}$. On $\left|y-y_{j}\right|=r$ in the parametric ball $V_{j}$ about $y_{j}(j=0,1),\left|\operatorname{grad}_{y} t_{0}(y)\right|^{2} \sim r^{2-2 n}$. Since $e^{t_{0}(y)}\left(1+e^{t_{0}(y)}\right)^{-2}<e^{-t_{0}(y)}$ and $t_{0}(y) \sim r^{2-n}$ on $r=\left|y-y_{0}\right|$,

$$
\lambda^{2}(y) \sim\left\{\exp \left(-r^{2-n}\right)\right\} r^{2-2 n} \sim\left\{\exp -\frac{1}{r^{n-2}}\left[1-(2-2 n) r^{n-2} \log r\right]\right\}
$$

holds on $r=\left|y-y_{0}\right|$. Since $-r^{n-2} \log r \rightarrow 0$ as $r \rightarrow 0$,

$$
\lambda^{2}(y) \sim \exp \left(-r^{2-n}\right)
$$

holds as $r=\left|y-y_{0}\right| \rightarrow 0$.

Similarly, in the vicinity of $y_{1}$ on $r=\left|y-y_{1}\right|$, 


$$
\begin{aligned}
\lambda^{2}(y) & \sim\left[\exp \left(-r^{2-n}\right)\right]\left[1+\exp \left(-r^{2-n}\right)\right]^{-2} r^{2-2 n} \\
& \sim\left[1+\exp \left(-r^{2-n}\right)\right]^{-2} \exp \left\{-\frac{1}{r^{n-2}}\left[1-(2-2 n) r^{n-2} \log r\right]\right\},
\end{aligned}
$$

where $t_{0}(y) \sim-r^{2-n}$ on $r=\left|y-y_{1}\right| . \quad$ As $r=\left|y-y_{1}\right| \rightarrow 0,\left[1+\exp \left(-r^{2-n}\right)\right]^{-2}$ $\rightarrow 1$. Therefore, we have

$$
\lambda^{2}(y) \sim \exp \left(-r^{2-n}\right)
$$

as $r \rightarrow 0$ in $V_{y_{1}}$.

By virtue of Lemma $10, \lambda^{2}(y) d V_{y}$ can be continued to a non-negative finitely continuous $n$-form on $S$ and

$$
\Delta_{y} s(y, a)=\lambda^{2}(y)
$$

on $S-\{a\}$. Therefore we may regard $\lambda^{2}(y) d V_{y}$ as a volume element on $S$. Set $\omega(y)=\lambda^{2}(y) d V_{y}$. We will evaluate the total volume of $S$ with respect to $\omega(y)$.

In the case where $S$ is open, let $\left\{Q_{n}^{\prime}\right\}$ be a regular exhaustion of $S$. For a $C^{2}$-function $\varphi$ on $S$, we define the Dirichlet integral $D_{S}(\varphi)=D(\varphi)$ of $\varphi$ over $S$ as $\lim _{\Omega_{n}^{\prime} \rightarrow S} D_{\Omega_{n}^{\prime}}(\varphi)$ with $D_{\Omega_{n}^{\prime}}(\varphi)=\int_{\Omega_{n}^{\prime}} d \varphi \wedge * d \varphi$. Similarly we can define $\int_{S} \omega(y)=\lim _{\Omega_{n}^{\prime} \rightarrow S} \int_{\Omega_{n}^{\prime}} \omega(y)$. With these definitions we have;

LEMMA 11. For any $S$ which is either open or closed, the total volume of $S$ with respect to the volume element $\omega(y)$ is equal to 2 . In other words,

$$
\int_{S} \omega(y)=2
$$

Proof. Let $S$ be open and $\alpha_{j}(j=0,1)$ be the level hypersurface $\left\{y \in S: t_{0}(y)=r_{j}\right\} \quad(j=0,1)$. Set $D_{0}=\left\{y_{0}\right\} \cup\left\{t_{0}(y) \leq r_{0}\right\}$ and $D_{1}=\left\{y_{1}\right\} \cup$ $\left\{t_{0}(y) \geq r_{1}\right\}$. Then $D_{0}$ and $D_{1}$ are compact for sufficiently small $r_{0}$ and sufficiently large $r_{1}$. Moreover, by Sard's theorem, we can choose such a pair of $r_{0}$ and $r_{1}$ that $\alpha_{j}(j=0,1)$ contains no critical points of $t_{0}(y)$. For such a fixed pair of $r_{0}$ and $r_{1}$, let $\left\{\Omega_{n}^{\prime}\right\}$ be a regular exhaustion of $S$ with $\Omega_{n}^{\prime} \supseteq D_{0} \cup D_{1}$ for each $n$. By the same reason, we may assume that each $\partial \Omega_{n}^{\prime}$ contains no critical points of $t_{0}$. For any $r \in\left(r_{0}, r_{1}\right)$, set $\alpha\left(r, t_{0}\right)=\left\{y \in S: t_{0}(y)=r\right\}$ and $\alpha_{\Omega_{n}^{\prime}}\left(r, t_{0}\right)=\alpha\left(r, t_{0}\right) \cap \Omega_{n}$. Since $\int_{\partial \Omega_{n}^{\prime}} t_{0} * d t_{0} \rightarrow$ 0 as $\Omega_{n}^{\prime} \rightarrow S$ ([7]), Green's formula implies that 


$$
D_{S-D_{0} \cup D_{1}}\left(t_{0}\right)=\lim _{\Omega_{n}^{\prime} \rightarrow S} \int_{\alpha_{0}+\alpha_{1}} t_{0} * d t_{0}=r_{1}-r_{0} .
$$

Thus, by Fubini's theorem, we have

$$
\begin{aligned}
D_{S-D_{0} \cup D_{1}}\left(t_{0}\right) & =\lim _{\Omega_{n}^{\prime} \rightarrow S} \int_{\alpha_{\Omega_{n}^{\prime}}\left(r, t_{0}\right)}\left(\int_{r_{0}}^{r_{1}} d t_{0}\right) * d t_{0} \\
& =\left(r_{1}-r_{0}\right) \lim _{\Omega_{n}^{\prime} \rightarrow S} \int_{\alpha_{\Omega_{n}^{\prime}}\left(r, t_{0}\right)} * d t_{0} .
\end{aligned}
$$

These two equations imply that, for $r \in\left(r_{0}, r_{1}\right)$,

$$
\lim _{\Omega_{n}^{\prime} \rightarrow S} \int_{\alpha_{\Omega_{n}^{\prime}}\left(r, t_{0}\right)} * d t_{0}=1 \quad \text { a.e.. }
$$

This guarantees the caluculation;

$$
\begin{aligned}
\int_{S-D_{0} \cup D_{1}} \omega(y) & =\lim _{\Omega_{n}^{\prime} \rightarrow S} \int_{r_{0}}^{r_{1}} e^{r}\left(1+e^{r}\right)^{-2}\left\{\int_{\alpha_{\Omega_{n}^{\prime}}\left(r, t_{0}\right)} * d t_{0}\right\} d r \\
& =\left\{\left(1+e^{r_{1}}\right)^{-1}-\left(1+e^{r_{0}}\right)^{-1}\right\}
\end{aligned}
$$

By Lemma 10,

$$
\int_{D_{0}} \omega(y)=0\left(\left|r_{0}\right|\right) \text { and } \int_{D_{1}} \omega(y)=0\left(r_{1}\right) .
$$

Thus we conclude that

$$
\int_{S} \omega(y)=\lim _{\substack{r_{1} \rightarrow \infty \\ r_{0} \rightarrow-\infty}}\left\{\int_{S-D_{0} \cup D_{1}} \omega(y)+\int_{D_{0} \cup D_{1}} \omega(y)\right\}=2 .
$$

In the case where $S$ is compact, the number of critical points of $t_{0}$ are finite. Evaluating directly we have the same conclusion. Thus the proof is completed.

As a by-product we have;

COROLlaRY 2. In the case of a non-compact $S$, we have for $r \in(-\infty, \infty)$

$$
\lim _{\Omega_{n}^{\prime} \rightarrow S} \int_{\alpha_{\Omega_{n}^{\prime}}\left(r, t_{0}\right)} * d t_{0}=1 \quad \text { a.e.. }
$$

5. We are now ready to give the first main theorem according to Sario's method ([8], [9], [11], and [12]). We will state some notation and formulate the first main theorem in this section, while the proof shall be given in section 6 .

Let $f: R \rightarrow S$ be an arbitrary harmonic mapping between an open 
Riemannian $n$-space $R$ and a Riemannian $n$-space $S$. On $R$ choose a parametric ball $R_{0}$ with the border $\beta_{0}$. A subregion $\Omega$ of $R-\bar{R}_{0}$ will be called an adjacent regular region if $\Omega \cup \bar{R}_{0}$ is a regular region of $R$. Given such an $\Omega$. We form the harmonic function $u=u_{\Omega}$ in $\bar{\Omega}$ which vanishes on $\beta_{0}$ and is equal to a constant $k=k_{\Omega}$ and which satisfies $\int_{\beta_{0}} * d u=1$, where the constant $k=k_{\Omega}$ is determined by the harmonic modulus $e^{1 / k}$ of $\Omega$. As for the harmonic modulus we refer to [13].

For $h \in\left(0, k_{\Omega}\right]$, let $\beta_{h}$ be the level hypersurface of $u=h$ and $\Omega_{h}$ be the adjacent regular region $\left.u^{-1}(0, h)\right)$ and, for the given non-singular value $a \in S-\left\{y_{0}, y_{1}\right\}$ of $f$, let $\nu(h, a)$ be the number of the pre-images of $a$ in $\Omega_{h}$. We choose the A-function (counting function) as

$$
A(h, a)=\int_{0}^{h} \nu(r, a) d r .
$$

It reflects the frequency of the $a$-points. For the $B$-function (proximity function) we take

$$
B(h, a)=\int_{\beta_{h}-\beta_{0}} s(f(x), a) * d u .
$$

The integrand is the proximity of the image of $\beta_{h}-\beta_{0}$ to $a$ under $f$ and hence $B(h, a)$ represents the mean proximity of the image of $\beta_{h}-\beta_{0}$ to $a$.

As in section 4 we define

$$
\lambda^{2}(f(x))=e^{t_{0}(f(x))}\left(1+e^{t_{0}(f(x))}\right)^{-2}\left|\operatorname{grad}_{x} t_{0}(f(x))\right|^{2} .
$$

Then $\lambda^{2}(f(x)) d V_{x}$ with the locally Euclidian volume element $d V_{x}$ on $R$ is a non-negative finitely continuous $n$-form on $R$ by Lemma 10 and

$$
\Delta_{x} s(f(x), a)=\lambda^{2}(f(x))
$$

holds on $R-f^{-1}(a)$. We set $\omega(f(x))=\lambda^{2}(f(x)) d V_{x}$. This is independent of the point $a$, since $\omega(f(x))=0(1)$ at each point of $f^{-1}(a)$.

The $C$-function (characteristic function) $C(h)$ is defined as

$$
C(h)=\int_{0}^{h}\left\{\int_{R_{r}} \omega(f(x))\right\} d r,
$$

where $R_{r}=\Omega_{r} \cup \bar{R}_{0}$. The counting function and the proximity function are essentially determined by the point $a$ and $h$, where $h$ is determined by the region $\Omega_{h}$. But the characteristic function depends only on $\Omega_{h}$ 
and independent of $a$. We will see;

THEOREM. For any harmonic mapping $f$ of an open Riemannian $n$-space $R$ to a Riemannian $n$-space $S$ and for any non-singular value $a \in S$ of $f$,

$$
A\left(k_{\Omega}, a\right)+B\left(k_{\Omega}, a\right)=C\left(k_{\Omega}\right)
$$

holds.

We stress here that the $A-, B$-, and $C$-functions are function of adjacent regular region $\Omega$, not the scalors. The functions $u_{R}=\lim _{\Omega \rightarrow R} u_{\Omega}$ and $e^{1 / k}=e^{1 / \lim \Omega \rightarrow R} k_{\Omega}$ exist and the latter is referred to as the harmonic modulus of $R$. According as $k_{R}<\infty$ or $k_{R}=\infty, R$ is said to be hyperbolic or parabolic. Suppose that $R$ is hyperbolic. If $R$ is regular in the sence that $u_{R}^{-1}([0, h])$ is compact for each $h \in\left[0, k_{R}\right)$, then we can view that the $A-, B$-, and $C$-functions are functions on $\left[0, k_{R}\right)$ and our Theorem will be the proper generalization of the Nevanlinna first main theorem for the disk $|z|<1$. However, in general, $R$ is not regular and we have to take $\Omega$ as variables. If $R$ is parabolic, then the situation is much clearer. In this case we can make use of the Evans harmonic function $p(x)$ on $R$ instead of $u_{R}$. It is defined as follows (see [5] and [13]): $\mathrm{p}(x)$ is a positive harmonic function on $R-\bar{R}_{0}$ with $p \mid \beta_{0}=0, p=\infty$ at the ideal boundary $\beta$ of $R$ and $\int_{\beta_{0}} * d p=1$. Clearly $p^{-1}([0, h])$ is compact for each $h \in[0, \infty)$ and the $A-, B$-, and $C$-functions are functions on $[0, \infty)$, and our Theorem with this variation is the proper generalization of the Nevanlinna first main theorem for the plane $|z|<\infty$.

6. We will prove Theorem. Let $\left\{a_{j}\right\}$ be the pre-image of $a$ in $\Omega$ $=\Omega_{k}$. The set $\left\{a_{j}\right\} \cap \Omega_{h}$ has no accumulation points in $\bar{\Omega}_{h}$ for every $h \in[0, k)$, since $a$ is non-singular and $\left\{a_{j}\right\} \cap \Omega_{h}$ is compact in $\bar{\Omega}_{h}$. Without loss of generality we may assume that $\partial \Omega_{h}$ contains no $a$-points. In fact, since the only singularity of $s(f(x), a)$ is Newtonian, the hypersurface integrals of $s(f(x), a)$ that we shall consider will be finite and continuous in $h$ and our formulas will be extended to the case $a_{j} \in \partial \Omega_{h}$. For each $a_{j}$ in $\Omega_{h}$, choose a disjoint parametric ball $\Delta_{j}$ about $a_{j}$ which is contained in $\Omega_{h}$ and set $\alpha_{j}=\partial \Delta_{j}$. Applying Green's formula to the functions $v(x)=h-u(x)$ and $s(f(x), a)$, 


$$
\begin{gathered}
\int_{\beta_{1}-\beta_{0}-\Sigma \alpha_{j}} v(x)^{*} d s(f(x), a)-s(f(x), a)^{*} d v(x) \\
=\int_{\Omega_{h}-\cup \Delta_{j}} v(x)^{*} d s(f(x), a),
\end{gathered}
$$

where $\alpha_{j}$ and $\Delta_{j}$ run over all the $a$-points in $\Omega_{h}$. As $\alpha_{j}$ shrinkes to $a_{j}$, $\int_{\alpha_{j}} s(f(x), a)^{*} d v \rightarrow 0$ and, by Lemma $8, \int_{-\alpha_{j}} * d s\left(f(x), f\left(a_{j}\right)\right)=-\int_{\alpha_{j}} * d g_{\Delta_{j}}(x, a)$. Thus, in the shrinking process of $\alpha_{j} \rightarrow 0$,

$$
\begin{aligned}
\int_{-\Sigma \alpha_{j}} v(x)^{*} d s(f(x), a)-s(f(x), a)^{*} d v(x) \\
\quad=\int_{-\Sigma \alpha_{j}} v(x)^{*} d g_{d_{j}}\left(x, a_{j}\right) \rightarrow \sum v\left(a_{j}\right)=\int_{0}^{h}(h-r) d \nu(r, a) .
\end{aligned}
$$

The integration by part gives

$$
\int_{0}^{h}(h-r) d \nu(r, a)=-h \nu(0, a)+\int_{0}^{h} \nu(r, a) d r .
$$

Hence we have from (1)

$$
\begin{aligned}
& -h \nu(0, a)+\int_{0}^{h} \nu(r, a) d r+\int_{\beta_{h}-\beta_{0}} s(f(x), a)^{*} d u-h \int_{\beta_{0}} * d s(f(x), a) \\
& =\int_{\Omega_{h}} v(x) \omega(f(x)) .
\end{aligned}
$$

Again we apply Stokes' formula to the small region $R_{0}-\cup \Delta_{j}^{0}$ and the function $s(f(x), a)$, where the $\Delta_{j}^{0}\left(\subset R_{0}\right)$ are the small disjoint parametric balls about the $a$-points. Then, we have

$$
\int_{\beta_{0}} * d s(f(x), a)+\nu(0, a)=\int_{R_{0}} \omega(f(x)) .
$$

From this equation and (2),

$$
\int_{0}^{h} \nu(r, a) d r+\int_{\beta_{h}-\beta_{0}} s(f(x), a)^{*} d u=h \int_{R_{0}} \omega(f(x))+\int_{\Omega_{h}} v(x) \omega(f(x)) .
$$

Next we show that, for almost all $h \in[0, k]$, the $h$-derivative of the right hand side of (3) is the volume of $R_{h}$ with respect to $\omega(f(x))$. To show this it suffices to show that, for almost all $h \in[0, k]$,

$$
\frac{d}{d h}\left(\int_{\Omega_{h}}(h-u(x)) \omega(f(x))\right)=\int_{\Omega_{h}} \omega(f(x)) .
$$

For sufficiently small $\Delta h>0$, let $\Omega_{h+\Delta h}$ be the region with $\Omega_{h+\Delta h}=$ 
$\left\{x \in R-\bar{R}_{0}: u(x) \leqq h+\Delta h\right\}$. Since $0<h+\Delta h-u<\Delta h$ on $\Omega_{h+\Delta h}-\Omega_{h}$,

$$
\lim _{\Delta h \rightarrow+0} \int_{\Omega_{h+\Delta h-\Omega_{h}}} \frac{h+\Delta h-u}{\Delta h} \omega(f(x))=\lim _{\Delta h \rightarrow+0} \int_{\Omega_{h+\Delta h-\Omega_{h}}} \omega(f(x))=0 .
$$

Therefore

$$
\begin{aligned}
\lim _{\Delta h \rightarrow+0} & \frac{1}{\Delta h}\left[\int_{\Omega_{h+\Delta h}}(h+\Delta h-u) \omega(f(x))-\int_{\Omega_{h}}(h-u) \omega(f(x))\right] \\
= & \lim _{\Delta h \rightarrow+0} \int_{\Omega_{h+\Delta h-\Omega_{h}}} \frac{h+\Delta h-u}{\Delta h} \omega(f(x))+\int_{\Omega_{h}} \omega(f(x)) \\
= & \int_{\Omega_{h}} \omega(f(x))
\end{aligned}
$$

is valid for almost all $h \in[0, k]$ by virtue of Sard's theorem. Thus the derivative from the right of $\int_{\Omega_{h}}(h-u(x)) \omega(f(x))$ is equal to $\int_{\Omega_{h}} \omega(f(x))$ for almost all $h \in[0, k]$. A similar calculation holds for the derivative from the left. Therefore the equation (4) is valid for almost all $h \in[0, k]$ and we have

$$
h \int_{R_{0}} \omega(f(x))+\int_{\Omega_{h}}(h-u(x)) \omega(f(x))=\int_{0}^{h}\left[\int_{R_{r}} \omega(f(x))\right] d r .
$$

Combining the equations (4) and (5), the proof of Theorem is completed.

\section{REFERENCES}

[1] S. S. Chern: Complex analytic mappings of Riemann surfaces, Amer. J. Math., $82(1960), 323-337$.

[2] C. Constantinescu-A. Cornea: Compactifications of harmonic spaces, Nagoya Math. J., 25 (1965) , 1-57.

[ 3 ] S. Helgason: Differential geometry and symmetric spaces, Academic Press, 1962.

[4] H. Imai: Sario's potentials on Riemannian spaces, Pacific J. Math., 38 (1971), 441-455.

[5] M. Nakai: Infinite boundary value problems for second order elliptic partial differential equations, J. Fac. Sci. Univ. Tokyo, Sec. I. A., 17 (1970), 101-121.

[6 ] A. de la Pradelle: Approximation et caractère de quasi-analyticité dans la théore axiomatique des fonctions harmoniques, Ann. Inst. Fourier, Grenoble, 17 (1967), 383-399.

[ 7 ] B. Rodin-L. Sario: Principal functions, D. Van Nostland, 1967.

[ 8 ] L. Sario: Value distribution under analytic mappings of arbitrary Riemann surfaces, Acta Math., 109 (1963), 1-10.

[9] - : General value distribution theory, Nagoya Math. J., 23 (1963), 213-229.

[10] - : Second main theorem without exceptional intervals on Riemann surfaces, Michigan Math. J., 10 (1963), 1-10.

[11] — : A theorem on mappings into Riemann surfaces of infinite genus, Trans. Amer. Math. Soc., 117 (1965), 276-284. 
[12] L. Sario-K. Noshiro: The value distribution theory, D. Van Nostland, 1966.

[13] L. Satio-M. Nakai: Classification theory of Riemann surfaces, Springer-Verlag, 1970.

[14] H. Wu: Mappings of Riemann surfaces (Nevanlinna theory), Proc. Sympos. Pure Math. vol. XI, Entire functions and related parts of analysis. Amer. Math. Soc. 1968, 480-532.

[15] — : The equidistribution theory of holomorphic curves, Ann. of Math. Studies No. 64 (1970), Princeton.

Daido Institute of Technology 